\title{
Decomposition analysis on the macroeconomic drivers of the carbon emission from energy consumption in China
}

\author{
Junsong Jia ${ }^{1,2, a}$, Huiyong Jian ${ }^{1,2,3, b}$, Min $\mathrm{Ju}^{1,2, \mathrm{c}}$ and Dongming $\mathrm{Xie}^{4, \mathrm{~d}}$ \\ ${ }^{1}$ Key Laboratory of Poyang Lake Wetland and Watershed Research, Ministry of Education, Jiangxi \\ Normal University, Nanchang, Jiangxi, 330022, China \\ ${ }^{2}$ School of Geography and Environment, Jiangxi Normal University, Nanchang, Jiangxi, 330022, \\ China \\ ${ }^{3}$ School of Graduate, Jiangxi Normal University, Nanchang, Jiangxi, 330022, China \\ ${ }^{4}$ Jiangxi Science \& Technology Normal University, Nanchang, Jiangxi, 330013, China \\ ajiaaniu@126.com, bgoogjobjian2008@163.com, ‘gjxsddljm@126.com, djxstnu_xdm@jxstnu.edu.cn
}

Keywords: industries, carbon emission (CE), energy consumption (EC), LMDI, China.

Abstract. To make clear the relationship between the macroeconomic driving factors and the carbon emission (CE) of energy consumption (EC) in China, we first computed this CE during 2000-2014 and then analyzed the corresponding drivers by the Logarithmic Mean Divisia Index (LMDI) method. The results show the economic scale effect was the biggest driver. Its promoting effect was $12.69 \mathrm{Bt}$ and the corresponding contribution was 163\% during 2000-2014. The energy intensity's effect was the second largest but negative driver, with the mitigating effect of $4.6 \mathrm{Bt}$ and inhibiting contribution of 59\%, respectively. However, the effects of industrial structure and energy mix were very small, although all negative (mitigating). The energy mix was the last one, with the mitigating effect (contribution) of only $0.09 \mathrm{Bt}(1 \%)$. From three different periods (2000-2005, 2005-2010 and 2010-2014), the industrial structure effects (contributions) were $0.13 \mathrm{Bt}(5 \%),-0.06 \mathrm{Bt}(-2 \%)$ and $-0.59 \mathrm{Bt}(-22 \%)$, respectively. This indicated the related policies of industries' structural adjustment have started to put up an obvious (despite of not big) impact on reducing the EC and corresponding CE in China, especially in 2010-2014. The similar results can also be gotten from the point view of the three different industries, especially in the secondary industry. So, based on these, some scientific policy suggestions for the low carbon and sustainable development in China were recommended.

\section{Introduction}

With the rapid development of economy and society, more and more fossil fuels (or energies) are used in the lives. So the corresponding carbon emission (CE) from energy consumption (EC) is, also, increasingly emitted into the sky [1]. If we do not take any effective actions, the world's average temperature will increase about $5.8^{\circ} \mathrm{C}$ during the next 100 years [2]. This poses a huge threat to global sustainable development [3]. Thus, we can easily conclude that it is necessary for us to study the CE and its relative drivers more thoroughly in order to bring forward some efficient measures for solving this problem.

China, the world's largest developing country, has already become one of the top energy consumer as well as the top $\mathrm{CO}_{2}$ emitter in the world [3]. It was reported that China had discharged $7.9 \mathrm{Gt}^{\mathrm{CO}_{2}}$ in 2012, which was the biggest share and about a quarter of worldwide emissions at that time $[4,5]$. Since 2008, it has contributed about $80 \%$ for the world's rise of CE [7,8]. Therefore, it is an essential and the key work to more deeply study the $\mathrm{CE}$ and the corresponding drivers in China.

In the other sides, the Logarithmic Mean Divisia Index (LMDI) model has been considered as a perfect method [8], because it has some outstanding properties such as strong theoretical foundation (e.g., no unexplained residuals), the high adaptability, the ease of use, and so on.

So, this LMDI model mentioned above was chosen in this paper, based on the data availability and the problem's importance, for analyzing the CE of the EC in the years of 2000-2014 in China. 


\section{Data and methods}

Data sources: The data of EC and CE estimation are from China Energy Statistics Yearbook, China Statistics Yearbook [6] and relevant bulletin, etc.

Method of CE estimation: The method used in this study is from the article [7].

LMDI model: Based on the article [2], the LMDI model can be expressed as the follows. First, the $\mathrm{CE}$ is abbreviated as $C$ in the Eq. 1 and it is considered as the accumulation of four parameters:

$$
C=\sum_{i=1}^{3} C_{i}=\sum_{i=1}^{3}\left(Y \times \frac{Y_{i}}{Y} \times \frac{E_{i}}{Y_{i}} \times \frac{C_{i}}{E_{i}}\right)
$$

where $C$ denotes the total $\mathrm{CE}$; $C i$ denotes the $i$ industry's $\mathrm{CE} ; Y$ denotes the Gross Domestic Product (GDP); $Y_{i}$ denotes the $i$ industry's GDP; $E_{i}$ denotes the $i$ industry's energy consumption; $i=1,2$ and 3. They denote the primary industry (Pri.), the secondary industry (Sec.) and the tertiary industry (Ter.), respectively, which are divided by the relevant department of the national statistic's authority [6]. In Eq. 1, we suppose:

$$
S_{i}=\frac{Y_{i}}{Y}, I_{i}=\frac{E_{i}}{Y_{i}}, F_{i}=\frac{C_{i}}{E_{i}}
$$

So, the Eq. 1 can be expressed as:

$$
C=\sum_{i=1}^{3}\left(Y \times S_{i} \times I_{i} \times F_{i}\right)
$$

According to the article [7], the total $C$ change ( $\Delta C_{\text {tot }}$ ) between t years (target) and 0 years (base year) can be divided into the four effects of economies' scale $(\Delta C Y)$, industrial structure $(\Delta C s)$, energy intensity $\left(\Delta C_{I}\right)$ and energy $\operatorname{mix}\left(\Delta C_{F}\right)$ :

$$
\Delta C_{\text {tot }}=C_{T}-C_{0}=\Delta C_{Y}+\Delta C_{S}+\Delta C_{I}+\Delta C_{F}
$$

where the $\Delta C_{x}$ can be expressed as the follows (here, $x$ denotes the tot, $Y, S, I$ and $F$ in Eq. 4):

$$
\begin{aligned}
\Delta C_{x} & =\sum_{i=1}^{3} \omega_{i} \times \ln \frac{x_{i, T}}{x_{i, 0}} \\
\omega_{i} & =\frac{C_{i, T}-C_{i, 0}}{\ln C_{i, T}-\ln C_{i, 0}}
\end{aligned}
$$

\section{Results and analysis}

Fig. 1 shows the dynamics changes of the GDP, EC, EC's intensity (EC's amount per unit of GDP, EI), the total and different industries' CE in 2000-2014 in China. We can easily see the GDP was $10.03 \times 10^{12} \mathrm{RMB}$ in 2000 . It grew quickly up to $64.39 \times 10^{12} \mathrm{RMB}$ in 2014 , with an annual average growth amount of $3.88 \times 10^{12} \mathrm{RMB}$ and increasing rate of $14.21 \%$ (Fig. 1a), respectively.

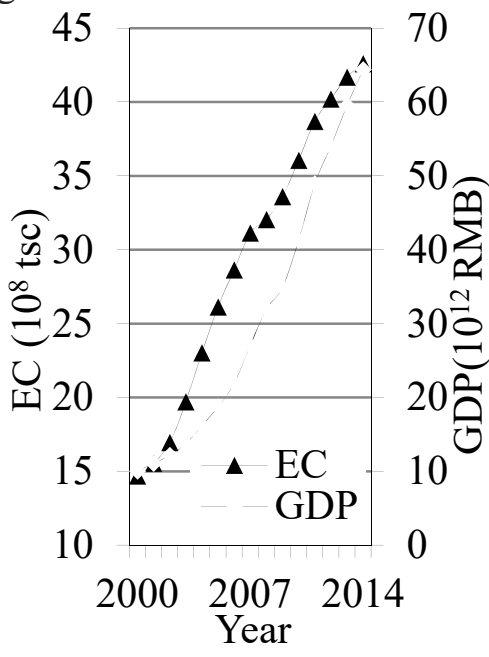

(a) GDP and EC

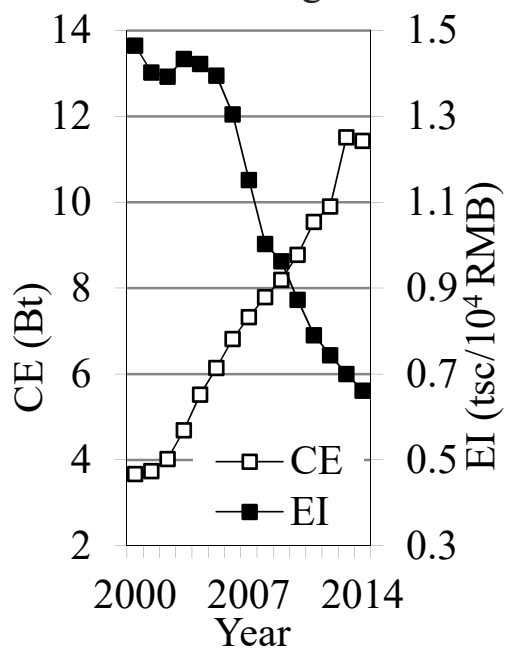

(b) $\mathrm{CE}$ and $\mathrm{EI}$

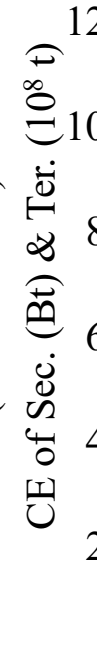

(c) CE of the Pri., Sec. and Ter.

Fig. 1 Dynamics changes of the GDP and EC, EI, total and different industries' CE in 2000-2014. 
Similarly, the EC has the whole increasing trend. It was $14.70 \times 10^{8}$ tonnes of standard coal (tsc) in 2000 and increased fast to $42.60 \times 10^{8}$ tsc in 2014 . The annual average growth amount was $1.99 \times 10^{8}$ tsc and the increasing rate was $7.90 \%$ (Fig. 1a).

However, the EI has a whole decrease trend as shown in Fig. 2b. In 2000, it was 1.47 tsc $/ 10^{4} \mathrm{RMB}$. In 2014, it decreased to 0.66 tsc $/ 10^{4} \mathrm{RMB}$. The annual average decline amount was $0.06 \mathrm{tsc} / 10^{4} \mathrm{RMB}$ and the decreasing rate was $5.74 \%$ (Fig. $1 \mathrm{~b}$ ). This is consistent with the results of many relevant studies [3, 7-8].

The CE has also a whole increase. It was 3.68 billion tonnes (Bt) in 2000 and $11.44 \mathrm{Bt}$ in 2014, with an annual average growth amount of $0.55 \mathrm{Bt}$ and rate of $8.4 \%$ (Fig. 1b), respectively. The total growth of the CE is $7.76(=11.44-3.68)$ Bt during 2000-2014, which can also be seen in Fig. $2 \mathrm{a}(\Delta$ Ctot).
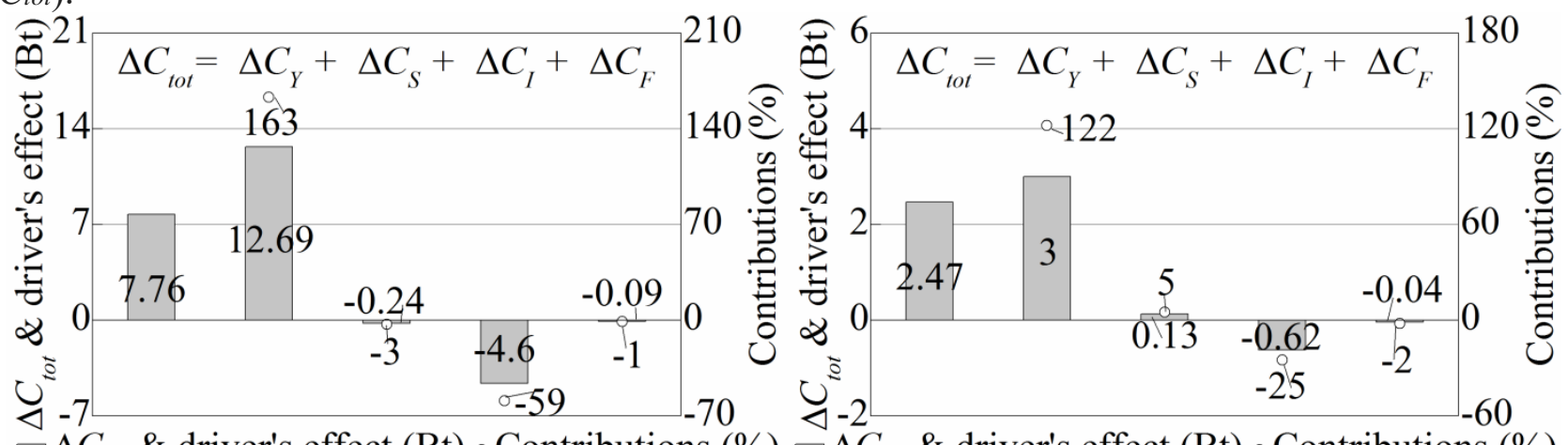

$\square \Delta C_{t o t}$ \& driver's effect (Bt) $\circ$ Contributions (\%) $\square \Delta C_{t o t}$ \& driver's effect (Bt) $\circ$ Contributions (\%) (a) 2000-2014

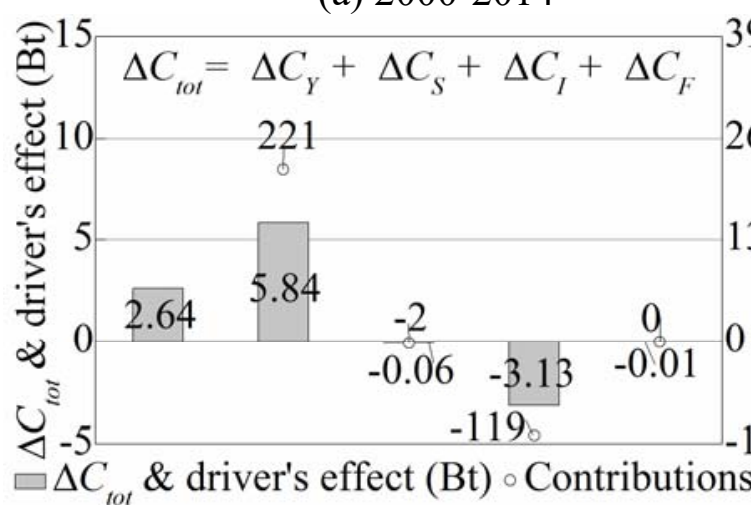

(c) 2005-2010

(b) 2000-2005

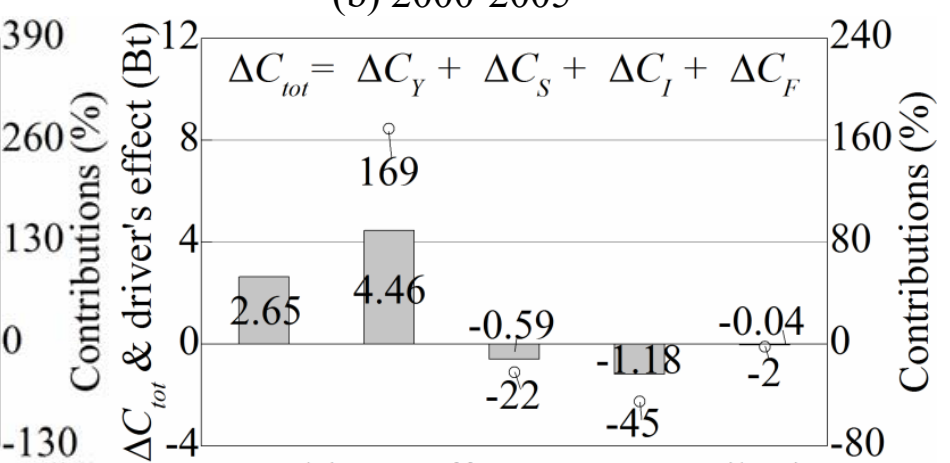

$(\%) \square \Delta C_{t o t} \&$ driver's effect $(\mathrm{Bt}) \circ$ Contributions $(\%)$

(d) 2010-2014

Fig. 2 The $\Delta C_{\text {tot }}$, the decomposition results of drivers and their corresponding contributions in different periods: (a) 2000-2014; (b) 2000-2005; (c) 2005-2010; (d) 2010-2014.

As shown in Fig. 2, the $\Delta C_{\text {tot }}$, the decomposition results of drivers and their corresponding contributions in the whole study period and different three periods are drawn based on the LMDI model.

We could easily find that the economic scale effect $\left(\Delta C_{Y}\right)$ was the biggest driver, with the CE's promoting effect of $12.69 \mathrm{Bt}$ and the corresponding contribution of 163\% during 2000-2014, respectively (Fig. 2a). The second largest driver of $\Delta C_{\text {tot }}$ was the EI's effect $\left(\Delta C_{I}\right)$, which had inhibited the CE's increase of $4.6 \mathrm{Bt}$ and its contribution was $-59 \%$.

We divided the whole period (2000-2014) into three different periods (2000-2005, 2005-2010 and 2010-2014) to observe unceasingly their changes and further make clear the impacts and contributions of these macroeconomic drivers (Fig. 2b, c \& d).

In the three stages, the total CE's increases were 2.47, 2.64 and $2.65 \mathrm{Bt}$, respectively. So, it can be seen that it had a slight but not obvious growth. Similar to the whole driving effects (Fig. 1a), the $\Delta C_{Y}$ was still the biggest driver, and the $\Delta C_{I}$ was the second largest driver of $\Delta C_{\text {tot. }}$. The promoting effects (contributions) of the $\Delta C_{Y}$ were $3 \mathrm{Bt}(122 \%), 5.84 \mathrm{Bt}(221 \%)$ and $4.46 \mathrm{Bt}(169 \%)$, respectively, in the different stages. In the same way, the inhibiting effects (contributions) of the $\Delta C_{I}$ were $0.62 \mathrm{Bt}(25 \%)$, $3.13 \mathrm{Bt}(119 \%)$ and $1.18 \mathrm{Bt}(45 \%)$, respectively (Fig. 2b, c \& d). 
On the contrary, the effects of industrial structure $\left(\Delta C_{S}\right)$ and energy mix $\left(\Delta C_{F}\right)$ were very small, but all negative (mitigating), which indicated that we should also give enough attention to them. Between the two drivers, the impact of the $\Delta C_{F}$ effect was only $-0.09 \mathrm{Bt}$, and the correspondingly mitigating contribution was no more than $1 \%$ (Fig. 2a).

In the same way, the impact of the $\Delta C_{F}$ was always the least one, and the mitigating contribution was no more than $2 \%$ in the different three periods (Fig. $2 b, c \& d$ ).

However, the impact effects (contributions) of the $\Delta C s$ were $0.13 \mathrm{Bt}(5 \%),-0.06 \mathrm{Bt}(-2 \%)$ and $-0.59 \mathrm{Bt}(-22 \%)$, respectively, in different three stages. This indicated the related policies of industries' structural adjustment have started to put up an obvious impact on reducing the EC and corresponding $\mathrm{CE}$ under the strategy of national macroeconomic development, especially in recent years.

Therefore, to further identify the carbon reduction reason and find the main potential of the industries' structural adjustment, the drivers' results and the corresponding contributions from the different industries (Pri., Sec. and Ter.) in the three periods were analyzed as shown in Table 1.

Table 1 . Drivers results $\left(10^{7} \mathrm{t}\right)$ and the corresponding contributions $(\%$, numbers in the upper right of the brackets) from different industries in three periods.

\begin{tabular}{c|ccc|ccc|ccc}
\hline \multirow{2}{*}{ Var. } & \multicolumn{3}{|c|}{$2000-2005$} & \multicolumn{3}{c|}{$2005-2010$} & \multicolumn{3}{c}{$2010-2014$} \\
\cline { 2 - 9 } & Pri. & Sec. & Ter. & Pri. & Sec. & Ter. & Pri. & Sec. & Ter. \\
\hline$\Delta C_{Y}$ & $5.8^{(2.4)}$ & $261.5^{(106)}$ & $32.7^{(13)}$ & $7.2^{(2.7)}$ & $519^{(197)}$ & $57.2^{(21.7)}$ & $3.9^{(1.5)}$ & $398.8^{(150.3)}$ & $43.6^{(16.4)}$ \\
$\Delta C_{S}$ & $-2.2^{(-0.9)}$ & $13.4^{(5)}$ & $2^{(1)}$ & $-1.8^{(-0.7)}$ & $-8.8^{(-3)}$ & $4.6^{(1.8)}$ & $-0.4^{(0)}$ & $-66.1^{(-25)}$ & $8^{(3)}$ \\
$\Delta C_{I}$ & $-0.9^{(-0.3)}$ & $-40.1^{(-16)}$ & $-21.5^{(-9)}$ & $-8.5^{(-3.2)}$ & $-266^{(-101)}$ & $-38.7^{(-15)}$ & $-1^{(-0.1)}$ & $-92.3^{(-35)}$ & $-25^{(-9.6)}$ \\
$\Delta C_{F}$ & $-0.1^{(-0.1)}$ & $-6.5^{(-3)}$ & $2.7^{(1)}$ & $-0.1^{(-0.1)}$ & $-2.6^{(-1)}$ & $1.8^{(0.8)}$ & $-0.2^{(0)}$ & $-4.2^{(-1.6)}$ & $0.2^{(0.1)}$ \\
\hline$\Delta C_{\text {tot }}$ & $2.6^{(1.1)}$ & $228.3^{(92)}$ & $15.8^{(6)}$ & $-3.2^{(-1.3)}$ & $241.6^{(92)}$ & $24.9^{(9.3)}$ & $2.3^{(1.4)}$ & $236.3^{(88.7)}$ & $26.8^{(9.9)}$ \\
\hline
\end{tabular}

On the whole, the secondary industry was the main contributor (Table 1). The accumulating effects (contributions) of all the macroeconomic drivers from this industry were $2.28 \mathrm{Bt}(92 \%), 2.42$ $\mathrm{Bt}(92 \%)$ and $2.36 \mathrm{Bt}(89 \%)$, respectively, in the different three periods (Table 1). The reason may be arising from the industrial characteristics in itself, which had the strongest increasing trend than the primary and the tertiary industries (Fig. 1c).

Particularly, the effects (contributions) of $\Delta C_{S}$ from the secondary industry were $0.13 \mathrm{Bt}(5 \%)$, $-0.09 \mathrm{Bt}(-3 \%)$ and $-0.66 \mathrm{Bt}(-25 \%)$, respectively, in the different three periods (Table 1). The results were consistent with the above and indicated the related policies of industries' structural adjustment, especially in the secondary industry, had the important impact on reducing the EC and corresponding CE.

\section{Conclusion}

Using the available data of the related EC and macroeconomic indicators in 2000-2014 in China, we, first, computed the $\mathrm{CE}$ and observed the dynamics changes of these variables and found there were close influencing effects from these indicators to the CE.

Thus, to make clear the relationship between them, we analyzed this problem by using the LMDI method. The results showed the economic scale effect was the biggest driver, with the promoting effect of $12.69 \mathrm{Bt}$ and contribution of $163 \%$ during 2000-2014, respectively. The energy intensity 's effect was the second largest but negative driver. Its mitigating effect was $4.6 \mathrm{Bt}$, with a correspondingly inhibiting contribution of $59 \%$.

However, the effects of industrial structure and energy mix were very small, but all negative (mitigating). Among them, the energy mix was the least one. Its mitigating effect was only $0.09 \mathrm{Bt}$, with a negative contribution of no more than $1 \%$.

From three different periods (2000-2005, 2005-2010 and 2010-2014), we can find the industrial structure effects (contributions) were $0.13 \mathrm{Bt}(5 \%),-0.06 \mathrm{Bt}(-2 \%)$ and $-0.59 \mathrm{Bt}(-22 \%)$, respectively. This indicated the related policies of industries' structural adjustment have started to put up an obvious (despite of not big) impact on reducing the EC and corresponding CE in China, especially in recent years. 
To further identify the carbon reduction reason of the industries' structural adjustment, the drivers' results and the corresponding contributions from the different industries (Pri., Sec. and Ter.) were analyzed. The results similar with the above, especially in the secondary industry, were gotten. Namely, the related policies of industries' structural adjustment, especially in the secondary industry, had the important impact on reducing the EC and corresponding CE.

In the end, based on these findings, we could bring forward some concrete suggestions and countermeasures for the low carbon and sustainable development in China. For example, we should carry out unceasingly the scientific and reasonable adjustment of industrial structure, to improve energy use efficiency and reduce the corresponding $\mathrm{CE}$, such as promoting the rate of industries with low fuel consumptions and high added value, and gradually passing out these industries with backward fuel efficiencies, etc.

\section{Acknowledgements}

The aids of the Fund of National Natural Science (71473113, 31360120, 51408584) and Natural Science Foundation of Jiangxi (20151BAB203040) are gratefully acknowledged. Corresponding author Junsong Jia can be contacted by (86)18607918843 or jiaaniu@,126.com.

\section{References}

[1] Q.W. Wang, B. Su, J. S. Sun, P. Zhou and D. Q. Zhou: Measurement and decomposition of energy-saving and emissions reduction performance in Chinese cities. Applied Energy, 2015, 151(8): 85-92.

[2]J.W. Sun: Changes in energy consumption and energy intensity: a complete decomposition model. Energy Economics, 1998, 20(1): 85-100.

[3] J.M. Cansino, R. Roman and M. Ordonez: Main drivers of changes in $\mathrm{CO}_{2}$ emissions in the Spanish economy: A structural decomposition analysis. Energy Policy, 2016, 89(2): 150-159.

[4] B.W. Ang and F.L. Liu: A new energy decomposition method: perfect in decomposition and consistent in aggregation. Energy, 2001, 26(6): 537-548.

[5] J. Albrecht, D. Francois and K. Schoors: A shapley decomposition of carbon emissions without residuals. Energy Policy, 2002, 30(9): 727-736.

[6] Information on http://www.stats.gov.cn/tjsj/ndsj/.

[7] L. Liu, S.S. Wang, K. Wang, R.Q. Zhang and X.Y. Tang: LMDI decomposition analysis of industry carbon emissions in Henan province, China: Comparison between different 5-years plans. Natural Hazards, 2016, 80(2):997-1014.

[8] S. Ren, X. Fu and X. H. Chen: Regional variation of energy-related industrial $\mathrm{CO}_{2}$ emissions mitigation in China. China Economic Review, 2012, 23(4):1134-1145.

[9] B. Xiao, D. Niu and X. Guo: The drivers of changes in $\mathrm{CO}_{2}$ emissions in China: A structural decompostion analysis. Energies, 2016, 9(4), 259-269.

[10] M. Zhang, X. Liu, W. Wang and M. Zhou: Decomposition analysis of CO2 emissions from electricity generation in China. Energy Policy, 2013, 52(2), 159-165. 